\title{
Relativistic Correction of the Rydberg Formula
}

\author{
Koshun Suto \\ Chudai-Ji Temple, Isesaki, Japan \\ Email: Koshun_suto129@mbr.nifty.com
}

How to cite this paper: Suto, K. (2020) Relativistic Correction of the Rydberg Formula. Journal of Modern Physics, 11, 294-303.

https://doi.org/10.4236/jmp.2020.112018

Received: January 10, 2020

Accepted: February 18, 2020

Published: February 21, 2020

Copyright (c) 2020 by author(s) and Scientific Research Publishing Inc. This work is licensed under the Creative Commons Attribution International License (CC BY 4.0).

http://creativecommons.org/licenses/by/4.0/

\section{(c) (i) Open Access}

\begin{abstract}
The relationship $E=-K$ holds between the energy $E$ and kinetic energy $K$ of the electron constituting a hydrogen atom. If the kinetic energy of the electron is determined based on that relationship, then the energy levels of the hydrogen atom are also determined. In classical quantum theory, there is a formula called the Rydberg formula for calculating the wavelength of a photon emitted by an electron. In this paper, in contrast, the formula for the wavelength of a photon is derived from the relativistic energy levels of a hydrogen atom derived by the author. The results show that, although the Rydberg constant is classically a physical constant, it cannot be regarded as a fundamental physical constant if the theory of relativity is taken into account.
\end{abstract}

\section{Keywords}

Rydberg Formula, Rydberg Constant, Classical Quantum Theory, Energy-Momentum Relationship in a Hydrogen Atom, Relativistic Kinetic Energy

\section{Introduction}

In the classical quantum theory of Bohr, the energy levels of the hydrogen atom are given by the following formula [1] [2].

$$
\begin{aligned}
E_{\mathrm{BO}, n} & =-\frac{1}{2}\left(\frac{1}{4 \pi \varepsilon_{0}}\right)^{2} \frac{m_{\mathrm{e}} e^{4}}{\hbar^{2}} \cdot \frac{1}{n^{2}} \\
& =-\frac{\alpha^{2} m_{\mathrm{e}} c^{2}}{2 n^{2}}, \quad n=1,2, \cdots .
\end{aligned}
$$

Here, $E_{\text {во }}$ refers to the total mechanical energy predicted by Bohr. Also, $\alpha$ is the following fine-structure constant.

$$
\alpha=\frac{e^{2}}{4 \pi \varepsilon_{0} \hbar c} .
$$


Bohr thought the following quantum condition was necessary to find the energy levels of the hydrogen atom.

$$
m_{\mathrm{e}} v_{n} \cdot 2 \pi r_{n}=2 \pi n \hbar .
$$

The energy of the hydrogen atom is also given by the following formula.

$$
E=-K=\frac{1}{2} V(r)=-\frac{1}{2} \frac{1}{4 \pi \varepsilon_{0}} \frac{e^{2}}{r} .
$$

If $E$ in Equation (1b) is substituted into Equation (4), then the following formula can be derived as the orbital radius of the electron.

$$
r_{n}=4 \pi \varepsilon_{0} \frac{\hbar^{2}}{m_{\mathrm{e}} e^{2}} n^{2}, \quad n=1,2, \cdots
$$

The photonic energy emitted during a transition between energy levels $\left(E_{\mathrm{BO}, n}-E_{\mathrm{BO}, m}\right)$ and wavelength $\lambda$ for principal quantum numbers $m$ and $n$ can be expressed as follows.

$$
E_{\mathrm{BO}, n}-E_{\mathrm{BO}, m}=h v=\frac{h c}{\lambda}=h c R_{\infty}\left(\frac{1}{m^{2}}-\frac{1}{n^{2}}\right), m=1,2, \cdots, n=m+1, m+2, \cdots .
$$

Here, $R_{\infty}$ is the Rydberg constant, which is defined by the following equation.

$$
R_{\infty}=\frac{\alpha^{2} m_{\mathrm{e}} c}{2 h}=\frac{2 \pi^{2} m_{\mathrm{e}} e^{4}}{c h^{3}} .
$$

The Rydberg formula can be derived from Equation (6) as indicated below.

$$
\frac{1}{\lambda}=\frac{E_{\mathrm{BO}, n}-E_{\mathrm{BO}, m}}{h c}=R_{\infty}\left(\frac{1}{m^{2}}-\frac{1}{n^{2}}\right), \quad m=1,2, \cdots, \quad n=m+1, m+2, \cdots .
$$

\section{Relationship Enfolded in Bohr's Quantum Condition}

This section to Section 4 are excerpts from another paper, but this material is repeated because it is needed here. The Planck constant $h$ can be written as follows [3]:

$$
\hbar=\frac{h}{2 \pi}=\frac{m_{\mathrm{e}} c \lambda_{\mathrm{C}}}{2 \pi} .
$$

Here, $\lambda_{\mathrm{C}}$ is the Compton wavelength of the electron.

When Equation (9) is used, the fine-structure constant $\alpha$ can be expressed as follows.

$$
\alpha=\frac{e^{2}}{2 \varepsilon_{0} m_{\mathrm{e}} c^{2} \lambda_{\mathrm{C}}} .
$$

Also, the classical electron radius $r_{\mathrm{e}}$ is defined as follows.

$$
r_{\mathrm{e}}=\frac{e^{2}}{4 \pi \varepsilon_{0} m_{\mathrm{e}} c^{2}} .
$$

If $r_{\mathrm{e}} / \alpha$ is calculated here,

$$
\frac{r_{\mathrm{e}}}{\alpha}=\frac{\lambda_{\mathrm{C}}}{2 \pi}
$$


If Equation (5) is written using $r_{\mathrm{e}}$ and $\alpha$, the result is as follows.

$$
r_{n}=4 \pi \varepsilon_{0} \frac{\hbar^{2}}{m_{\mathrm{e}} e^{2}} n^{2}=\frac{e^{2}}{4 \pi \varepsilon_{0} m_{\mathrm{e}} c^{2}}\left(\frac{4 \pi \varepsilon_{0} \hbar c}{e^{2}}\right)^{2} n^{2}=\frac{r_{\mathrm{e}}}{\alpha^{2}} n^{2} .
$$

Next, if $\hbar$ in Equation (9) and $r_{n}$ in Equation (13) are substituted into Equation (3),

$$
m_{\mathrm{e}} v_{n} \cdot 2 \pi \frac{r_{\mathrm{e}}}{\alpha^{2}} n^{2}=2 \pi n \frac{m_{\mathrm{e}} c \lambda_{\mathrm{C}}}{2 \pi} .
$$

If Equation (12) is also used, then Equation (14) can be written as follows.

$$
m_{\mathrm{e}} v_{n} \cdot 2 \pi \frac{r_{\mathrm{e}}}{\alpha^{2}} n^{2}=2 \pi n \frac{m_{\mathrm{e}} c r_{\mathrm{e}}}{\alpha} .
$$

From this, the following relationship can be derived [4].

$$
\frac{v_{n}}{c}=\frac{\alpha}{n} .
$$

\section{The Relation between Kinetic Energy and Momentum Derived from the STR Relationship}

The energy-momentum relationship in the special theory of relativity (STR) holds in an isolated system in free space. Here, if $m_{0}$ is rest mass and $m$ relativistic mass, the relationship can be written as follows.

$$
\left(m_{0} c^{2}\right)^{2}+p^{2} c^{2}=\left(m c^{2}\right)^{2} .
$$

What is the relationship between relativistic kinetic energy and momentum if this relationship holds?

Incidentally, Sommerfeld once defined kinetic energy as the difference between the relativistic energy $m c^{2}$ and rest mass energy $m_{0} c^{2}$ of an object [5]. That is,

$$
K=m c^{2}-m_{0} c^{2}=m_{0} c^{2}\left[\frac{1}{\left(1-\beta^{2}\right)^{1 / 2}}-1\right], \quad \beta=\frac{v}{c} .
$$

Sommerfeld believed that Equation (18), which can be derived from Equation (17), can also be applied to the electron in a hydrogen atom.

First, it is clear that the following formula holds [4].

$$
\left[m_{0} c^{2}+\left(m c^{2}-m_{0} c^{2}\right)\right]^{2}=\left(m c^{2}\right)^{2} \text {. }
$$

Expanding the left side of this equation yields the following.

$$
m_{0}^{2} c^{4}+\left(m^{2} c^{4}-m_{0}^{2} c^{4}\right)=\left(m_{0} c^{2}\right)^{2}+\left(m+m_{0}\right)\left(m c^{2}-m_{0} c^{2}\right) c^{2} .
$$

Using this, Equation (19) becomes as follows.

$$
\left(m_{0} c^{2}\right)^{2}+\left(m+m_{0}\right)\left(m c^{2}-m_{0} c^{2}\right) c^{2}=\left(m c^{2}\right)^{2} .
$$

Since this equation and Equation (17) are equal, the following relationship must hold when Equation (18) is taken into account. 


$$
p^{2}=\left(m_{0}+m\right)\left(m c^{2}-m_{0} c^{2}\right)=\left(m_{0}+m\right) K .
$$

The following formula is obtained from this.

$$
K_{\mathrm{re}}=\frac{p_{\mathrm{re}}^{2}}{m_{0}+m} .
$$

Here, $K_{\mathrm{re}}$ is relativistic kinetic energy and $p_{\text {re }}$ relativistic momentum. The "re" in $K_{\text {re }}$ and $p_{\text {re }}$ stands for "relativistic".

Equation (23) is the formula for relativistic kinetic energy. Classical (non-relativistic) kinetic energy, in contrast, is defined as follows.

$$
K_{\mathrm{cl}}=\frac{1}{2} m_{0} v^{2}=\frac{p_{\mathrm{cl}}^{2}}{2 m_{0}} .
$$

In classical theory, mass does not depend on velocity. That is, Equation (23) and Equation (24) are the same if $m=m_{0}$.

\section{Energy-Momentum Relationship of the Electron Derived with Another Method}

The author has previously derived the following relationships applicable to the electron constituting a hydrogen atom [6].

$$
\left(m_{\mathrm{e}} c^{2}\right)^{2}-p_{n}^{2} c^{2}=\left(m_{n} c^{2}\right)^{2}
$$

Here,

$$
m_{n} c^{2}=m_{\mathrm{e}} c^{2}-K_{\mathrm{re}, n} .
$$

$m_{n}$ is the mass of an electron in a state where the principal quantum number is $n$.

These energy relationships can be illustrated as follows (Figure 1).

In this paper, Equation (25) will be derived more simply by using a method different from that used previously. The logic of Equations (19) to (23) is borrowed to accomplish that purpose.

Now, it is clear that the following equation holds.

$$
\left[m_{n} c^{2}+\left(m_{\mathrm{e}} c^{2}-m_{n} c^{2}\right)\right]^{2}=\left(m_{\mathrm{e}} c^{2}\right)^{2}
$$

Expanding and rearranging this equation, the following equation is obtained.

$$
\left(m_{n} c^{2}\right)^{2}+\left(m_{\mathrm{e}}+m_{n}\right)\left(m_{\mathrm{e}} c^{2}-m_{n} c^{2}\right) c^{2}=\left(m_{\mathrm{e}} c^{2}\right)^{2} .
$$

Next, the relativistic kinetic energy of the electron can be defined as follows by referring to Equation (23).

$$
K_{\mathrm{re}, n}=m_{\mathrm{e}} c^{2}-m_{n} c^{2}=\frac{p_{\mathrm{re}, n}^{2}}{m_{\mathrm{e}}+m_{n}} .
$$

From this,

$$
\left(m_{\mathrm{e}}+m_{n}\right)\left(m_{\mathrm{e}} c^{2}-m_{n} c^{2}\right)=p_{\mathrm{re}, n}^{2} .
$$

Finally, Equation (28) matches Equation (25). 
Bohr's Theory

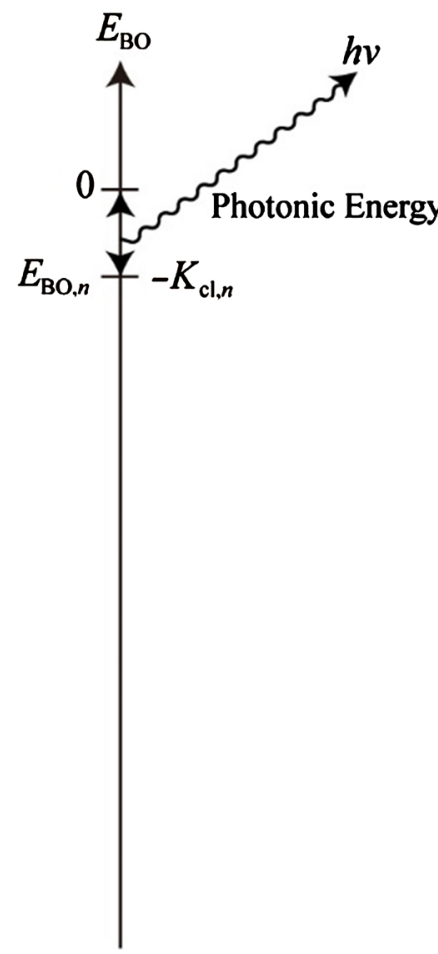

This Paper

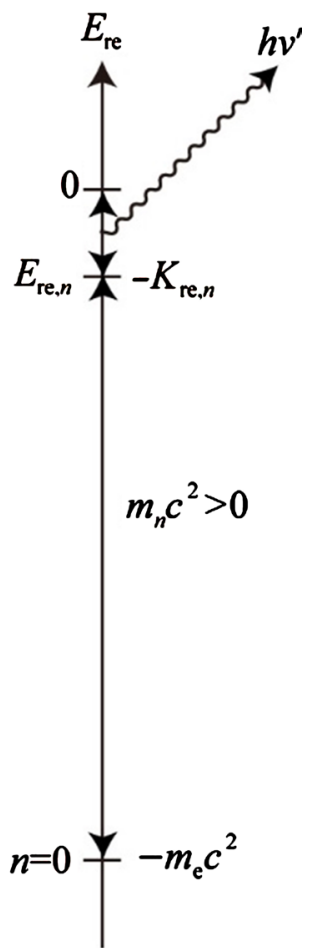

Figure 1. Energy levels of a hydrogen atom derived from Bohr's classical quantum theory and this paper: According to the virial theorem, $E_{\mathrm{BO}, n}=-K_{\mathrm{cl}, n}$ and $E_{\mathrm{re}, n}=-K_{\mathrm{re}, n}$. An electron at rest in free space emits a photon when it is taken into a hydrogen atom. Also, the electron acquires the same amount of kinetic energy as the energy of the emitted photon. If $E_{\mathrm{BO}}=0, E_{\mathrm{re}}=0$ are described using an absolute energy scale, then the electron is at rest in free space, and this corresponds to the state of having a rest mass energy of $m_{\mathrm{e}} c^{2}$.

Incidentally, $p_{\text {re }, n}=m_{n} v_{n}$ [4], and thus it is clear that the following equation holds.

$$
p_{\text {re }, n} c=m_{n} v_{n} c \text {. }
$$

Here, if we substitute $p_{\mathrm{re}, n} c$ in Equation (31) into Equation (25) and rearrange, then the following value is obtained.

$$
m_{n}=m_{\mathrm{e}}\left(1+\frac{v_{n}^{2}}{c^{2}}\right)^{-1 / 2}
$$

If the relation in Equation (16) is used here, Equation (32) becomes as follows.

$$
m_{n}=m_{\mathrm{e}}\left(1+\frac{\alpha^{2}}{n^{2}}\right)^{-1 / 2}=m_{\mathrm{e}}\left(\frac{n^{2}}{n^{2}+\alpha^{2}}\right)^{1 / 2} .
$$

Hence, the energy levels of a hydrogen atom $E_{\mathrm{re}, n}$ are:

$$
E_{\mathrm{re}, n}=-K_{\mathrm{re}, n}=m_{n} c^{2}-m_{\mathrm{e}} c^{2}=m_{\mathrm{e}} c^{2}\left[\left(1+\frac{\alpha^{2}}{n^{2}}\right)^{-1 / 2}-1\right], \quad n=0,1,2, \cdots .
$$




\section{Relativistic Energy of a Hydrogen Atom Derived from Equation (16)}

When both sides of Equation (16) are squared, and then multiplied by $m_{\mathrm{e}} / 2$,

$$
\frac{1}{2} \frac{m_{\mathrm{e}} v_{n}^{2}}{c^{2}}=\frac{1}{2} \frac{m_{\mathrm{e}} \alpha^{2}}{n^{2}} \text {. }
$$

Hence,

$$
E_{\mathrm{BO}, n}=-\frac{1}{2} m_{\mathrm{e}} v_{n}^{2}=-\frac{\alpha^{2} m_{\mathrm{e}} c^{2}}{2 n^{2}} .
$$

If Equation (16) is taken as a departure point, the energy levels of the hydrogen atom derived by Bohr can be derived immediately. Equation (16) has tremendous power. However, from a relativistic perspective, (1/2) $m_{\mathrm{e}} v_{n}^{2}$ is an approximation of the kinetic energy of the electron. Therefore, the energy in Equation (1) is also an approximation of the true value.

Next, let's try to derive the energy levels in the hydrogen atom from Equation (16). If both sides of Equation (16) are first squared, and then both sides are multiplied by $m_{n}^{2} /\left(m_{\mathrm{e}}+m_{n}\right)$.

$$
\frac{m_{n}^{2} v_{n}^{2}}{m_{\mathrm{e}}+m_{n}}=\frac{\alpha^{2} c^{2}}{n^{2}} \frac{m_{n}^{2}}{m_{\mathrm{e}}+m_{n}} .
$$

Here, the left side of Equation (37) is the relativistic kinetic energy of the electron, and thus the energy levels are:

$$
E_{\mathrm{re}, n}=-K_{\mathrm{re}, n}=-\frac{m_{n}^{2} v_{n}^{2}}{m_{\mathrm{e}}+m_{n}}=-\frac{\alpha^{2} c^{2}}{n^{2}} \frac{m_{n}^{2}}{m_{\mathrm{e}}+m_{n}} .
$$

Next, if Equation (33) is taken into account, the right side of Equation (38) is as follows.

$$
E_{\mathrm{re}, n}=-\frac{\alpha^{2} c^{2}}{n^{2}} \times\left(\frac{n^{2} m_{\mathrm{e}}^{2}}{n^{2}+\alpha^{2}}\right) \times \frac{1}{m_{\mathrm{e}}\left[1+\left(\frac{n^{2}}{n^{2}+\alpha^{2}}\right)^{1 / 2}\right]} .
$$

Next, the numerator and denominator of Equation (39) are multiplied by:

$$
1-\left(\frac{n^{2}}{n^{2}+\alpha^{2}}\right)^{1 / 2}
$$

When this is done, Equation (39) is as follows.

$$
\begin{aligned}
E_{\mathrm{re}, n} & =-\frac{\alpha^{2}}{n^{2}}\left(\frac{n^{2}}{n^{2}+\alpha^{2}}\right) m_{\mathrm{e}} c^{2} \times\left(\frac{n^{2}+\alpha^{2}}{\alpha^{2}}\right)\left[1-\left(\frac{n^{2}}{n^{2}+\alpha^{2}}\right)^{1 / 2}\right] \\
& =m_{\mathrm{e}} c^{2}\left[\left(\frac{n^{2}}{n^{2}+\alpha^{2}}\right)^{1 / 2}-1\right] \\
& =m_{\mathrm{e}} c^{2}\left[\left(1+\frac{\alpha^{2}}{n^{2}}\right)^{-1 / 2}-1\right] .
\end{aligned}
$$


Equation (34) can also be derived taking Equation (16) as a starting point. The discussion thus far in this section has provided an explanation by quoting another paper.

Now, if a Taylor expansion is performed on the right side of Equation (34),

$$
\begin{aligned}
E_{\mathrm{re}, n} & =m_{\mathrm{e}} c^{2}\left[\left(1-\frac{\alpha^{2}}{2 n^{2}}+\frac{3 \alpha^{4}}{8 n^{4}}-\frac{5 \alpha^{6}}{16 n^{6}}+\cdots\right)-1\right] \\
& =-m_{\mathrm{e}} c^{2}\left(\frac{\alpha^{2}}{2 n^{2}}-\frac{3 \alpha^{4}}{8 n^{4}}+\frac{5 \alpha^{6}}{16 n^{6}}-\cdots\right) \\
& =-\frac{\alpha^{2} m_{\mathrm{e}} c^{2}}{2 n^{2}}\left(1-\frac{3 \alpha^{2}}{4 n^{2}}+\frac{5 \alpha^{4}}{8 n^{4}}-\cdots\right) .
\end{aligned}
$$

Comparing Equation (41c) and Equation (1b), it is evident that Equation (1) is an approximation of Equation (34). That is,

$$
E_{\mathrm{re}, n} \approx E_{\mathrm{BO}, n} \text {. }
$$

Next, Table 1 summarizes the energies of a hydrogen atom obtained from Equation (1) and Equation (34) [7].

The following values of CODATA were used when calculating energies.

$$
\begin{gathered}
\alpha=7.2973525693 \times 10^{-3} . \\
c=2.99792458 \times 10^{8} \mathrm{~m} \cdot \mathrm{s}^{-1} . \\
m_{\mathrm{e}}=9.1093837015 \times 10^{-31} \mathrm{~kg} .
\end{gathered}
$$

The results derived from section 3 to 5 are summarized here in Table 2 .

In deriving the energy levels of a hydrogen atom, Sommerfeld began from Einstein's energy-momentum relationship. However, that is a mistake. The Einstein relation that holds in an isolated system in free space is not applicable in the space inside a hydrogen atom where there is potential energy. The author derived, for the first time, Equation (25) that is applicable to an electron in a hydrogen atom.

\section{Discussion}

In the sections up to the previous section, the groundwork was laid for finding a formula for the wavelength of a photon emitted from a hydrogen atom.

The differences in energy between different energy levels in the hydrogen atom can be found with the following formula.

Table 1. Comparison of the energies of a hydrogen atom predicted by Bohr's classical quantum theory and this paper.

\begin{tabular}{ccc}
\hline & Bohr's Energy Levels & This Paper \\
\hline$n=1$ & $-13.60569 \mathrm{eV}$ & $-13.60515 \mathrm{eV}$ \\
2 & $-3.40142 \mathrm{eV}$ & $-3.40139 \mathrm{eV}$ \\
3 & $-1.511744 \mathrm{eV}$ & $-1.511737 \mathrm{eV}$
\end{tabular}


Table 2. Formulas and energies derived from the standpoint of STR and Sommerfeld, and formulas and energies derived by the author: Equations marked at the right with an asterisk are quoted from Reference [4]. When discussed by depicting a classical picture, like the Bohrmodel, the electron moving within the atom becomes lighter as its velocity increases.

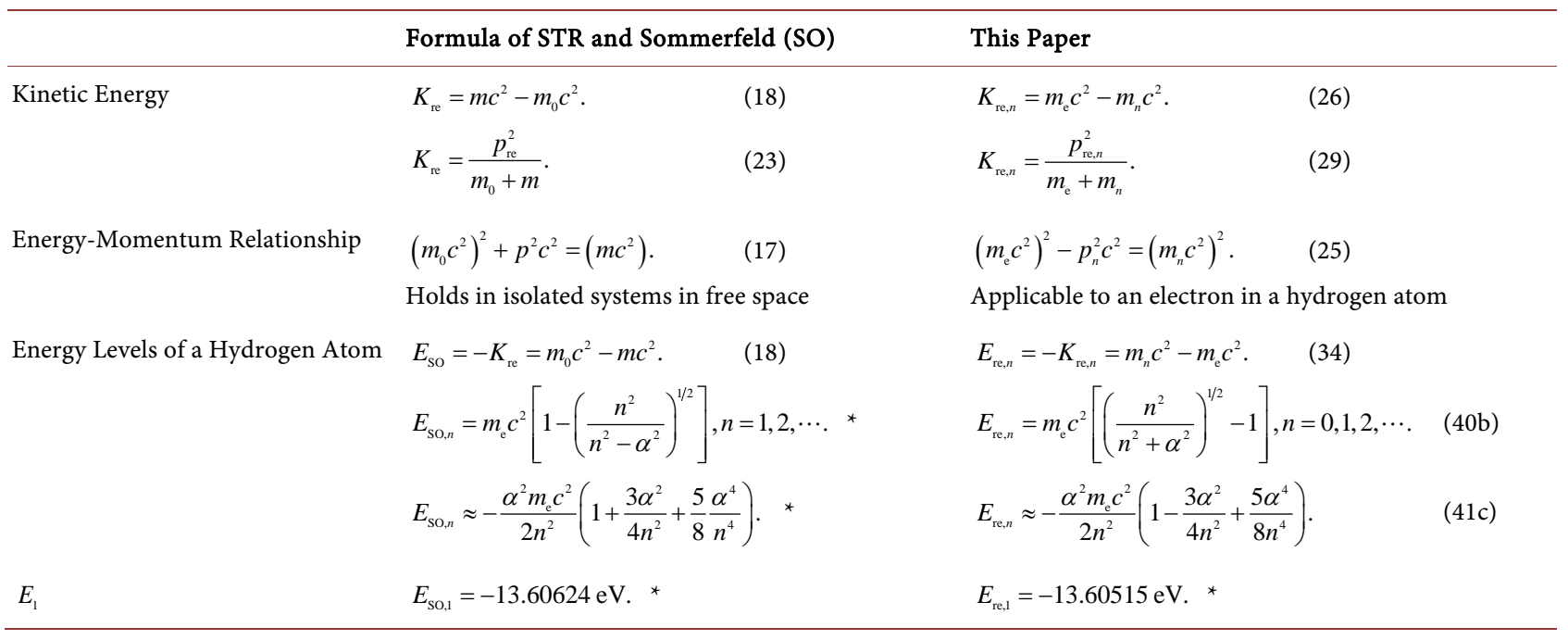

$$
\begin{aligned}
E_{\mathrm{re}, n}-E_{\mathrm{re}, m} & =\left(m_{n} c^{2}-m_{\mathrm{e}} c^{2}\right)-\left(m_{m} c^{2}-m_{\mathrm{e}} c^{2}\right)=m_{n} c^{2}-m_{m} c^{2}=h v=h \frac{c}{\lambda} \\
& =m_{\mathrm{e}} c^{2}\left[\left(1+\frac{\alpha^{2}}{n^{2}}\right)^{-1 / 2}-\left(1+\frac{\alpha^{2}}{m^{2}}\right)^{-1 / 2}\right], \quad n=m+1, m+2, \cdots
\end{aligned}
$$

The following equation is also known.

$$
\lambda_{\mathrm{C}}=\frac{h}{m_{\mathrm{e}} c} .
$$

Taking into account Equation (46),

$$
m_{\mathrm{e}} c^{2}=\frac{h c}{\lambda_{\mathrm{C}}} .
$$

Based on this, Equation (45) can be written as follows.

$$
\begin{aligned}
\frac{1}{\lambda} & =\frac{E_{\mathrm{re}, n}-E_{\mathrm{re}, m}}{h c} \\
& =\frac{1}{\lambda_{\mathrm{C}}}\left[\left(1+\frac{\alpha^{2}}{n^{2}}\right)^{-1 / 2}-\left(1+\frac{\alpha^{2}}{m^{2}}\right)^{-1 / 2}\right], m=0,1,2, \cdots, n=m+1, m+2, \cdots .
\end{aligned}
$$

Equation (48) is the formula for wavelength, taking into account Equation (25). If the Taylor expansion of Equation (48) is taken, the following formula is obtained.

$$
\begin{aligned}
\frac{1}{\lambda} & =\frac{1}{\lambda_{\mathrm{C}}}\left[\left(1-\frac{\alpha^{2}}{2 n^{2}}+\frac{3 \alpha^{4}}{8 n^{4}}-\frac{5 \alpha^{6}}{16 n^{6}}+\cdots\right)-\left(1-\frac{\alpha^{2}}{2 m^{2}}+\frac{3 \alpha^{4}}{8 m^{4}}-\frac{5 \alpha^{6}}{16 m^{6}}+\cdots\right)\right] \\
& =\frac{\alpha^{2}}{2 \lambda_{\mathrm{C}}}\left[\left(\frac{1}{m^{2}}-\frac{3 \alpha^{2}}{4 m^{4}}+\frac{5 \alpha^{4}}{8 m^{6}}-\cdots\right)-\left(\frac{1}{n^{2}}-\frac{3 \alpha^{2}}{4 n^{4}}+\frac{5 \alpha^{4}}{8 n^{6}}-\cdots\right)\right]
\end{aligned}
$$


Table 3. Wavelengths of photons emitted due to transitions between different energy levels: the 3 values in the table, in order from the top, are the values found from Equation (8), the values found from Equation (48), and the actual measured values.

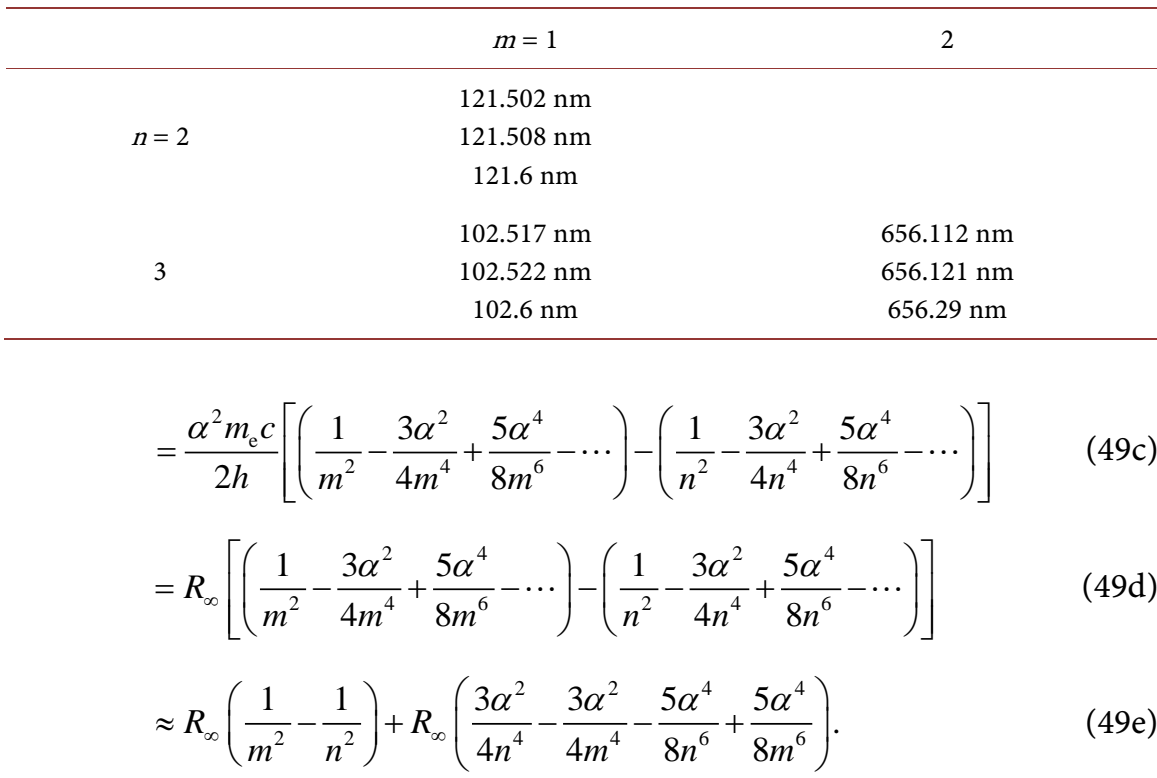

It is evident here that the approximation value of Equation (49e) matches the Rydberg formula Equation (8). That is,

$$
\frac{1}{\lambda_{\mathrm{C}}}\left[\left(1+\frac{\alpha^{2}}{n^{2}}\right)^{-1 / 2}-\left(1+\frac{\alpha^{2}}{m^{2}}\right)^{-1 / 2}\right] \approx R_{\infty}\left(\frac{1}{m^{2}}-\frac{1}{n^{2}}\right), n=m+1, m+2, \cdots
$$

Here, if the wavelengths of photons emitted due to transitions between different energy levels are calculated using Equations (8) and (48), the results are as indicated in Table 3.

Precision up to 4 significant digits is required for experiments. Therefore, there will be no problems even if the approximation of Equation (8) is used instead of Equation (48) to calculate wavelengths.

However, Equation (8) for calculating the wavelength of the spectra of a hydrogen atom is strange because it does not include the Compton wavelength of the electron.

\section{Conclusions}

In classical quantum theory, the wavelength of a photon emitted due to a transition by an electron to a different energy level is calculated using Equation (8). However, this paper has shown that Equation (48) is a formula more exact than calculating the wavelength of the photon.

Thus, it has been shown that the existing Equation (8) is an approximation for Equation (48).

\section{Acknowledgements}

I would like to express my thanks to the staff at ACN Translation Services for 
their translation assistance.

\section{Conflicts of Interest}

The author declares no conflicts of interest regarding the publication of this paper.

\section{References}

[1] Bohr, N. (1913) Philosophical Magazine, 26, 1. https://doi.org/10.1080/14786441308634955

[2] Bohr, N. (1952) Collected Works Vol. 2. North-Holland, Amsterdam, 136.

[3] Suto, K. (2015) Applied Physics Research, 7, 68-79. https://doi.org/10.5539/apr.v7n5p68

[4] Suto, K. (2019) Applied Physics Research, 11, 19-34. https://doi.org/10.5539/apr.v11n1p19

[5] Sommerfeld, A. (1923) Atomic Structure and Spectral Lines. Methuen \& Co. Ltd., London, 528.

[6] Suto, K. (2011) Physics Essays, 24, 301-307. https://doi.org/10.4006/1.3583810

[7] Suto, K. (2020) Journal of High Energy Physics, Gravitation and Cosmology, 6, 52-61. https://doi.org/10.4236/jhepgc.2020.61007 